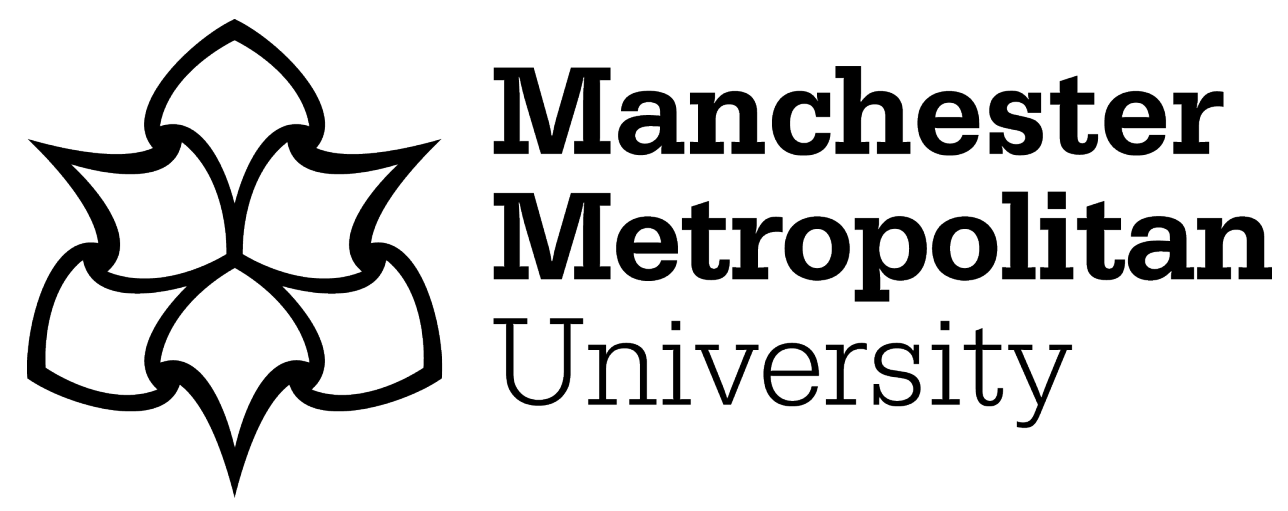

Lawton, R, Johnson, J, Janes, G ORCID logoORCID: https://orcid.org/00000002-1609-5898, Foy, R and Simms-Ellis, R (2019) Supporting doctors who make mistakes. The BMJ, 365. ISSN 0959-8146

Downloaded from: https: //e-space.mmu.ac.uk/625864/

Version: Published Version

Publisher: BMJ

DOI: https://doi.org/10.1136/bmj.I2161

Please cite the published version 


\section{Supporting doctors who make mistakes}

Rebecca Lawton, Judith Johnson, Gill Janes, Robbie Foy

Published BMJ 2019;365:I2161 doi: 10.1136/bmj.I2161 (Published 17 May 2019)

The term 'second victim' is provocative and, for those who have lost loved ones to medical error, it may seem an anathema. We agree that current systems often fail patients who are harmed and their families, not least by excluding them from investigations into what went wrong.

Our experience of 'second victim' research over the past decade highlights that the current adversarial approach does nothing to support learning and improvement. Rather it perpetuates the myth that some 'bad' doctors and nurses make mistakes; the rest are infallible. However, we found that $83 \%$ of doctors had been involved in a near-miss or adverse event at some point (Harrison et al., 2014). The uncomfortable truth is that most healthcare professionals make errors, and whether these lead to harm is often a matter of chance.

The authors argue that support systems are available for healthcare professionals, but these are only offered in a few forward-thinking organisations: only a third of UK physicians report adequate support from their organisation (Harrison et al., 2014). We (the NIHR Yorkshire and Humber Patient Safety Translational Research Centre) recently launched www.secondvictim.co.uk to address this gap, as well as start a dialogue with our patient involvement group about a sister website for patients and their families. Although we have received overwhelming support for this resource, we have identified a small number of concerns about the term 'second victim' and considered alternatives (e.g. 'second casualty.')

Language is powerful, and victim might imply a lack of accountability or responsibility. However, we have seldom, if ever, encountered a health professional who does not punish themselves for errors that harmed a patient. Unhelpful coping responses can lead to harmful behaviours, such as obsessive checking, ordering repeated tests and other defensive medicine practices, as well as adverse personal impacts. We suggest that, until better terminology is agreed, these consequences justify the term 'victim'. In an era where we are facing a workforce crisis (GMC, 2018) and a global shortage of healthcare workers (Britnell, 2019), a supportive stance towards professionals involved in errors is a cornerstone of high quality, sustainable healthcare delivery.

References

Harrison R, Lawton R, Stewart K. Doctors' experiences of adverse events in secondary care: the professional and personal impact. Clinical medicine. 2014 Dec 1;14(6):585-90.

General Medical Council (2018) "The state of medical education and practice in the UK", report.

Britnell M (2019) "Human: Solving the global workforce crisis in health care", Nuffield Trust guest comment. https://www.nuffieldtrust.org.uk/news-item/human-solving-the-global-workforce-crisisin-health-care 